\title{
Successful endoscopic management of a malignant gastroretroperitoneal fistula
}

Ali Alshati, MD, ${ }^{1}$ Mankanwal S. Sachdev, MD, FASGE, ${ }^{2}$ Alan Tan, MD,${ }^{3}$ Diego Muilenburg, MD, ${ }^{4}$ Toufic Kachaamy, $\mathbf{M D}^{5}$

Malignant involvement of the GI tract causing fistula is rare and can lead to infections, malnutrition, and poor quality of life, and can sometimes become life threatening. Surgical treatment can be challenging, especially in patients with severe malnutrition and poor functional status. Endoscopic management can improve the patient's nutrition, allowing

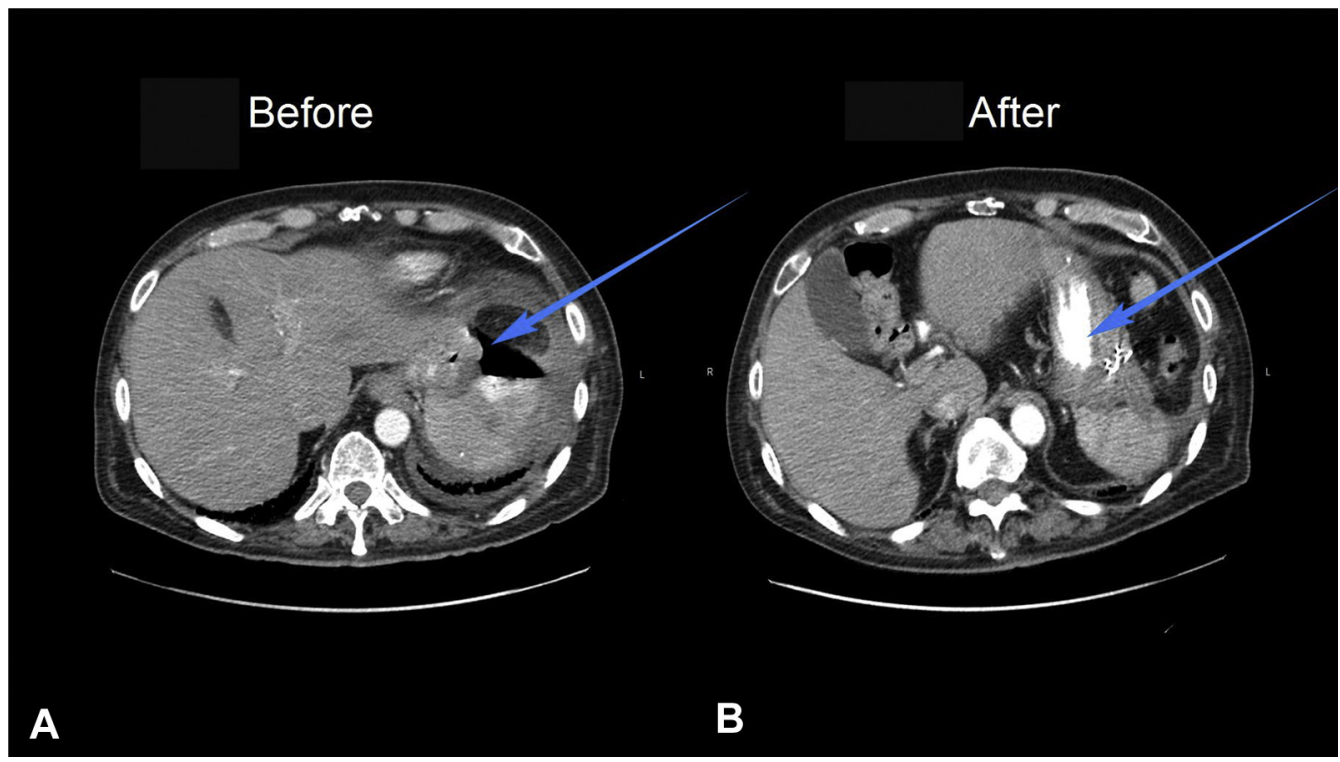

Figure 1. A, CT scan showing retroperitoneal mass and air, suggestive of a retroperitoneal abscess (arrow). B, CT scan showing resolution of the abscess and lack of extravasation of contrast material after fistula closure (arrow).
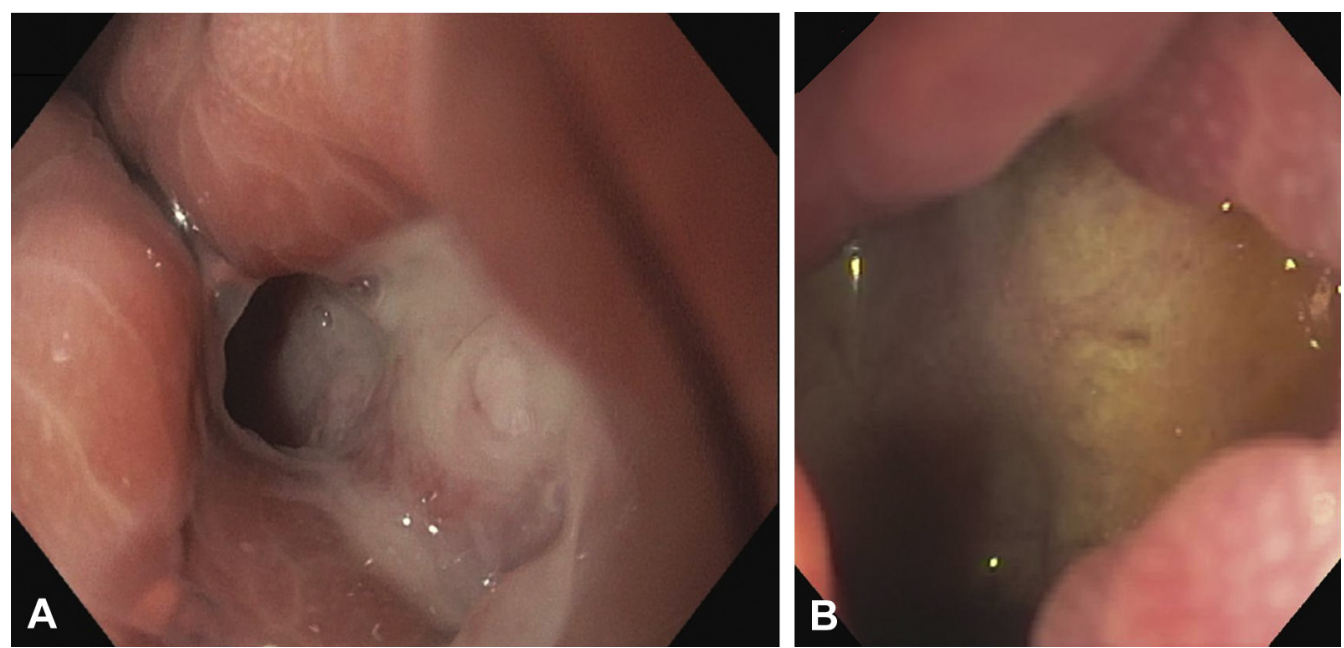

Figure 2. A, Endoscopic view showing small defect in the posterior wall of the stomach. B, Endoscopic view showing gastroretroperitoneal fistula and retroperitoneal abscess. 


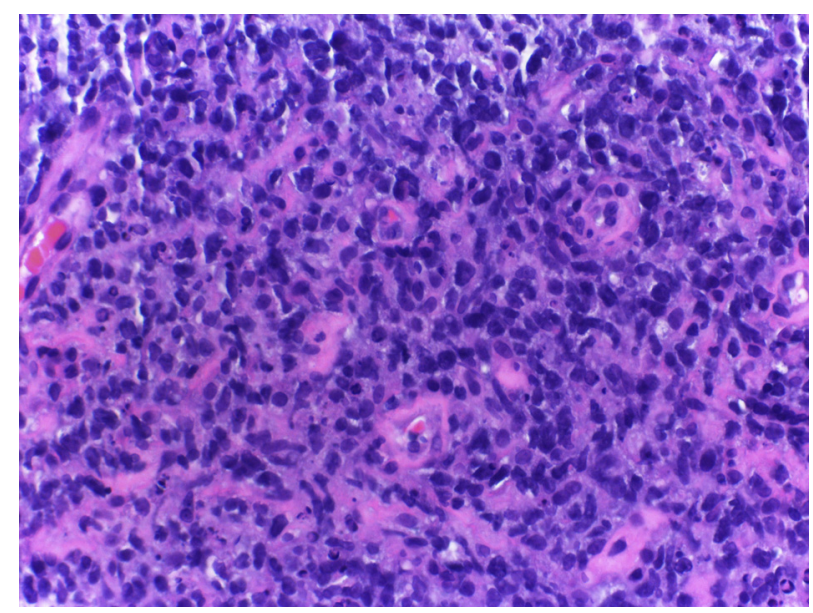

Figure 3. Biopsy specimen showing evidence of lymphoma. (H\&E, orig. mag. $\times 40)$.

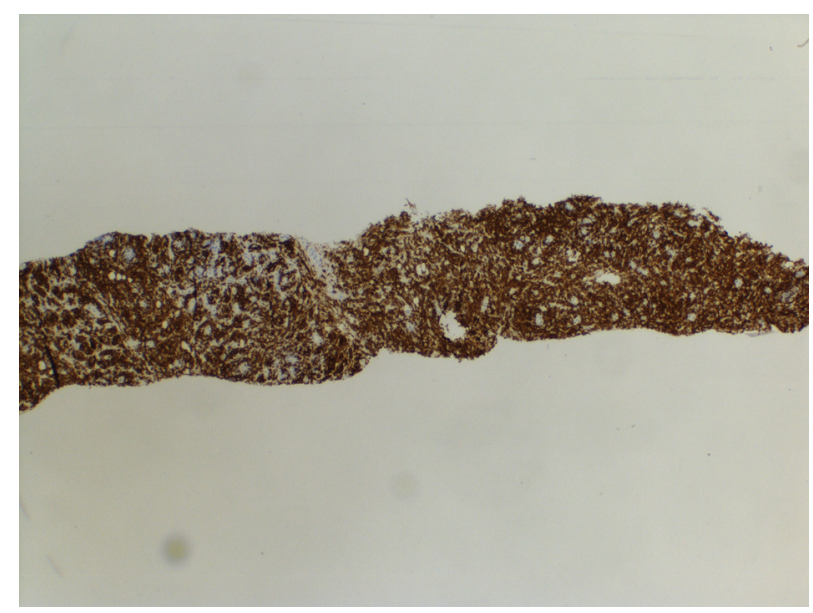

Figure 4. Biopsy specimen showing evidence of lymphoma. (Immunoperoxidase, orig. mag. $\times 10$ )

the patient to have definitive treatment, whether surgical or systemic therapy. We present a patient with gastric fistula due to involvement with lymphoma who was successfully treated endoscopically and then with systemic therapy (Video 1, available online at www.VideoGIE.org).

Primary gastric lymphoma is a rare tumor, accounting for $10 \%$ of lymphomas, $3 \%$ of gastric neoplasms, ${ }^{1}$ and $1 \%$ to $5 \%$ of gastric malignancies. ${ }^{2}$ Many patients present with advanced disease and adverse events such as upper-GI bleeding, obstruction, and, less commonly, perforation $(<2 \%)$, leading to fistula formation. Spontaneous gastric perforation in the absence of chemotherapy is extremely rare. EUS has become an indispensable tool for the diagnosis of gastric lymphomas. When EUS is combined with endoscopic biopsy, the overall accuracy approaches 90\% (vs 66\% for EUS alone).

A 55-year-old man presented with a few weeks' history of left-sided abdominal pain and a 22-pound weight loss. He appeared cachectic. Abdominal CT showed a retroperitoneal mass and abscess (Fig. 1A). There was extravasation of the oral contrast material into the abscess cavity, giving

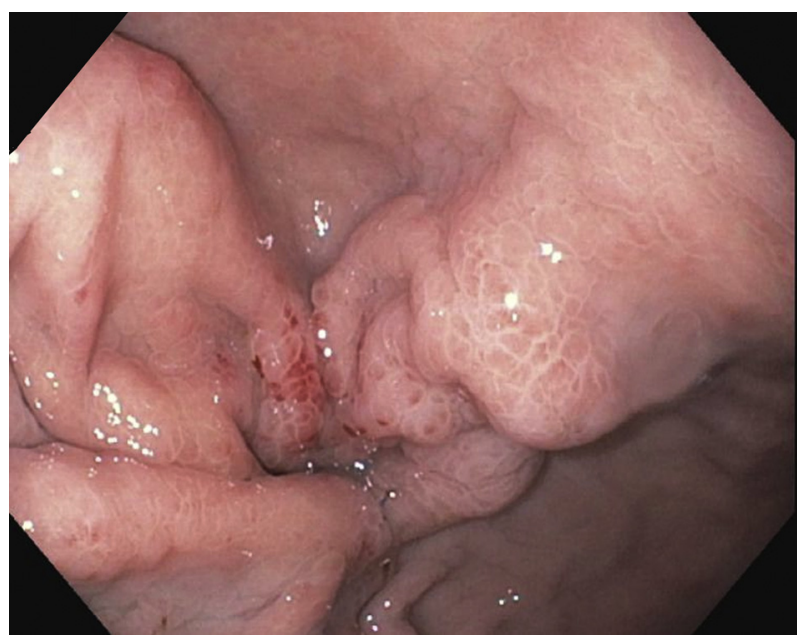

Figure 5. Endoscopic view showing final outcome of suturing.

a diagnosis consistent with gastric fistula. Endoscopy confirmed these findings (Fig. 2A and B). A biopsy specimen from the mass showed lymphoma (Figs. 3 and 4).

The patient was a poor surgical candidate, given his comorbidities, malnutrition, and poor functional status. He was treated in a multidisciplinary fashion, with percutaneous drainage of his abscess and endoscopic closure of his fistula, followed by systemic therapy. Endoscopic closure consisted of ablation of the fistula track with argon plasma coagulation, then multilayer suturing to close the gastric wall defect. A gastrojejunal tube was placed to divert the flow from the fistula tract and improve his nutrition. Three weeks later, endoscopy showed that the fistula had closed (Fig. 5). At that time, his functional and nutritional status had improved, allowing him to start chemotherapy. Follow-up CT 3 months later showed resolution of the abscess and no extravasation of contrast material (Fig. 1B). The gastrojejunal tube was removed, and the patient started to eat normally. He was disease free at the 5-month follow-up visit, and his functional status returned to baseline.

In a patient with a gastric fistula, neoplastic process should be considered if there is no prior surgery or other known risk factors, such as chemotherapy. Spontaneous gastric perforation is a rare adverse event of gastric lymphoma that is potentially life threatening.

This case illustrates the successful multidisciplinary management of a complex fistula in a patient who was a poor surgical candidate. Initial treatment focused on abscess drainage and nutritional support. The endoscopic intervention included argon plasma coagulation to ablate the mucosa at the edges of the fistula tract to create a larger submucosal surface, which was more likely to adhere together when approximated by suturing. Care was taken to make sure the entire surface was ablated. The OverStitch endoscopic suturing device (Apollo Endosurgery, Austin, Tex, USA) was used to approximate the edges and close the defect. Initially, a running layer of 
sutures was placed to close the fistula defect. Subsequently, 3 interrupted sutures were placed to lessen the tension from the first layer of sutures and to increase the likelihood of healing. By these steps, the multilayer suturing was completed, and the gastric wall defect was closed. This endoscopic intervention was accompanied by draining the underlying abscess, improving nutritional status, and treating the underlying malignancy.

To our knowledge, this endoscopic management for a lymphoma-induced gastric wall fistula is novel and has not been described in the literature before. Jiang et $\mathrm{al}^{4}$ reported a case of a successful example of multidisciplinary treatment of secondary aortoenteric fistula complicated by GI bleeding and retroperitoneal abscess. However, the management plan in that case included open laparotomy and multilevel surgical procedures. Pinsker et $\mathrm{al}^{5}$ described a successful endoscopic management of a persistent bronchobiliary fistula. The fistula in that case resulted from surgeries that included hemicolectomy and multiple bilobar liver wedge resections.

Endoscopy played a major role in the treatment of this patient. Endoscopic techniques and tools continue to evolve, making the therapeutic endoscopist more versatile, with an ever-expanding role in the management of complex GI conditions previously tackled by surgery.

\section{DISCLOSURE}

All authors disclosed no financial relationships relevant to this publication.

\section{REFERENCES}

1. Freeman C, Berg JW, Cutler SJ. Occurrence and prognosis of extranodal lymphomas. Cancer 1972;29:252-60.

2. Gossios K, Katsimbri P, Tsianos E. CT features of gastric lymphoma. Eur Radiol 2000;10:425-30.

3. Harada N, Wiersema M, Wiersema L. Endosonography guided fine needle aspiration biopsy (EUS FNA) in the evaluation of lymphadenopathy: staging accuracy of EUS FNA versus EUS alone [abstract]. Gastrointest Endosc 1997;45:AB31.

4. Jiang C, Chen X, Li J, et al. A case report of successful treatment of secondary aortoenteric fistula complicated with gastrointestinal bleeding and retroperitoneal abscess in an elderly patient. Medicine (Baltimore) 2018;97:e11055

5. Pinsker N, Papoulas M, Sodergren M, et al. Successful endoscopic management of a persistent bronchobiliary fistula with Histoacryl/Lipiodol mixture. Ann R Coll Surg Engl 2018;100:e73-7.

Department of Internal Medicine, Maricopa Integrated Health System, Creighton University, Phoenix, Arizona, USA (1), Arizona Center for Digestive Health, Gilbert, Arizona, USA (2), Department of Hematology and Oncology, Western Regional Medical Center, Cancer Treatment Centers of America, Goodyear, Arizona, USA (3), Department of Surgical Oncology, Western Regional Medical Center, Cancer Treatment Centers of America, Goodyear, Arizona, USA (4), Department of Gastroenterology and Advanced Endoscopy, Western Regional Medical Center, Cancer Treatment Centers of America, Goodyear, Arizona, USA (5).

Copyright (c) 2018 American Society for Gastrointestinal Endoscopy. Published by Elsevier Inc. This is an open access article under the CC BYNC-ND license (http://creativecommons.org/licenses/by-nc-nd/4.0/).

https://doi.org/10.1016/j.vgie.2018.11.008 